\title{
AN EFFECTIVE UNDESIRED CONTENT FILTRATION AND PREDICTIONS FRAMEWORK IN ONLINE SOCIAL NETWORK
}

\author{
Gyan Prakash, \\ Software Engineer, R\&D Department, \\ Vee Eee Technologies Solutions Pvt. Ltd, Chennai, India \\ gyanprakash.95@gmail.com \\ Nishant Saurav \\ Senior Software and Team Lead, \\ Oracle India Private Limited, Bangalore, India \\ nishant.saurav@oracle.com \\ Venkata Reddy Kethu \\ Associate Director-Projects, \\ Cognizant Technology Solutions, Hyderabad, India \\ venkatareddy.kethu@cognizant.com
}

\begin{abstract}
Online Social Networks (OSNs) is major prevalent interactive media in current days to divide, collective, and allocate an essential amount of human life messages. In OSNs, messages filtering can also be worn for a dissimilar, more reactive, meaning. It is happening because of the alternative of posting or remarking different posts on fastidious open/private locales, brought in General Messages. Messages separating can thusly be utilized to give clients the fitness to naturally control the messages composed on their messages, by sifting through disposed of messages. Facebook enables clients to state to post messages (i.e., companions, characterized gatherings of companions or companions of companions). To overcome the problems, the proposed mechanism implements an estimated automated framework, is defined Filtered Wall (FW), to filter discarded content from OSN user contents. The objective of paper is to utilize effective classification technique to avoid overpowered by unsuccessful messages. Content filtering can additionally misused for a disparate, more responsive for OSNs. The procedures outline of a framework gives adaptable substance based content filtering for OSNs, in light of ML strategy. It sets up the connections similarly with the condition of the expertise in content-based separating based personalization for OSNs down alongside web substances. The focal segments of the Filtered Wall scheme are the Content Based Messages Filtering (CBMF) and the Short Text Classifier essentials. Based on experimental evaluations, proposed method performs good precision, recall and F1 score on overall dataset.
\end{abstract}

Keywords: Online Social Network, Unwanted Content, Content Classification, Filtered Walls.

\section{INTRODUCTION}

Online Social Networks (OSNs) is mainly utilized to style interactive medium to share, compose, and disperse an essential amount of human living messages. Once a day, steady messages include the trading of a few sorts of substance, including free substance, picture, sound, and video messages. Facebook messages typical customer create 80 bits of focus every month, although more than 40 billion quantity of material (the web joins, news report blog 
sections, stories, accumulations, notes, photo, et cetera.) are spread every month. The huge and energetic soul of these messages delivers commence for the work of web content mining methodologies intended to anticipate significant messages from unified databases. To provide a dynamic valuable help as a main priority boggling and testing endeavors required in online social network supervision, in instance, get the chance to power or messages sifting. Messages shifting have been essentially hunting down what concerns printed archives and, extra as of late, web content.

Online Social Networks (OSNs) offers a very effective technique to keep up disposed of messages on client messages. For example, Facebook enables clients to see status that is worthy to embed messages in their messages while, there is no substance based inclinations are kept up, and in this manner it is not promising to maintain a strategic distance from undesired messages, for ask for political or scornful ones, it doesn't make a difference to the client who contributes the information in OSN [8]. Offering the administration is not only a state of utilizing prior described website content digging strategies for an alternate reason; relatively it includes for suggesting uncommonly delegated portrayal frameworks. The techniques offer ordinary arrangement strategies that have genuine outskirts since short messages don't give enough word events [9].

The proposed mechanism implements estimated automated mechanisms, is defined Filtered Wall (FW), to sift disposed of messages from OSN client content. The objective of the paper is to use powerful order instrument to evade overpowered by discarded messages. Contents filtering can similarly mishandle for a dissimilar, more responsive, reason for Online Social Networks. Content filtering can be utilized to provide customers ability to normally manage the messages formed on their individual messages. It uses content grouping methods [1] to consequently offer short content substance with an arrangement of classes. Huge attempts in constructing an incredible small text classifier (STC) are resolved in assessment with decision of a course in action to representation.

The framework offers a controlling rule layer using an adaptable dialect to recognize Filtering Rules (FRs), displays that what substances, ought not to appear on their messages. FRs can keep an assortment of various separating criteria that can be shared and custom fitted as indicated by the client necessities. The procedures outline of a framework gives adaptable substance based content filtering for OSNs, in light of ML strategy. It sets up the connections similarly with the condition of the expertise in content-based separating based personalization for OSNs down alongside web substances.

In the paper are proceeds as takes after: Section 2 discussed related works for understanding previous work. Section 3 explores proposed methodology with techniques details. Section 4 discusses about result of proposed mechanism with comparative analysis. Finally, Section 5 concludes overall work.

\section{RELATED WORK}

In [1] considered different techniques for short-based text classification of datasets. In [2], the technique evaluated the learning model and elicitation technique making pre-arranged messages. Arrangement of edges, gotten from endogenous assets of small messages, is fragile together with exogenous messages associated with the setting from which the messages begin. To the extent the information concern, the present technique neural learning based arrangement which is remains with content grouping [3]. In particular, the general short substance grouping framework chipped away at Radial Basis Function Networks 
(RBFN) developed capacities going about delicate classifiers, bearing loud messages and obscure classes.

Messages filtering frameworks are wanted to order a surge of energetically produced messages dispatched non-concurrently by a messages maker and present to the client those messages that are relied upon to fulfill his/her necessities. Concentrating on the OSN area, consideration in get to control and security insurance is genuinely later. Current work is fundamentally based on security protecting data mining methodologies, which is, ensuring data associated with the framework, i.e., relations/center points, as performing relational organization learn. The greater part of these models passes on get to control supplies as far as connections that the requestor must have with the supply holder. For it, one of the key fundamentals of our plan is the accessibility of a defense for the message substance to be broken by the sifting methods. It additionally communicates the sifting rules. In recognize, there is no one can get to the control models past referred to utilize the substance of the assets to actualize get to control.

The convenience of substance build filtering as for messages posted on OSN customer messages postures different troubles provided the small length of messages more remote than broad variety topics can be discussed. Short substance order has recognized the reliable gathering. In [4] endeavored to advance the order of short content strings upward a semi-managed learning approach in light of a blend of marked preparing information in addition to a minor quantity of unlabeled however correlated longer articles. The assurance is unsuitable in a field in small messages are not processing or fraction of longer semantically correlated records. An alternate progress is arranged by [5] that skirt the issue of blunder inclined component creation by receiving a measurable learning strategy that can make sensibly well without divide generation. It's is hardened classifiers in nature and don't effectively coordinate delicate, multiparticipation ideal models. The frameworks consider an enduring participation to programs a key trademark for characterizing adaptable strategy based personalization procedures.

As of late, the misusing classification component is tended to for customizing path in OSNs. For case, a grouping strategy has been created to mark short instant messages stay away from overwhelming clients of characterized benefits by crude information. The frameworks depicted Twitter and connect an arrangement of classifications with each tweet relating its substance. The client would then be able to appraisal just positive classes of tweets in light of his/her advantages. In [6] recommended a strategy, is defined FilmTrust creates Online Social Network belief affiliations and attribution messages customize access to website. Though, some frameworks don't propose a separating method layer. In segregation, separating arrangement dialect allows the area of FRs as per a differing quality of criteria which does not consider just the consequences of the characterization strategy additionally the dealings of the divider holder with other OSN client's messages on the client profile [7]. In[10] composed new techniques to dodge the security dangers, examination administrations for guaranteeing the respectability and accessibility of outsourced information to pick up accomplish computerized criminology and unwavering quality in a cloud domain.

\section{PROPOSED SYSTEM}

Proposed work is stimulated by the many get to models and related lead dialects and requirement strategies which have been produced so far for OSNs, as sifting imparts a few similitude's to get to control. Content filtering can be 
measured as a development of get the chance to manage, as it can be utilized similarly to shield objects from not suitable topics, and subjects from ignoble things. The better bit of get the chance to manage methods laid out consequently maintains topology in OSNs. The methodology utilizes a comparative proposal to stock the customers to FR applies. In any case, proposed filtering approach dialect broadens the dialects planned for get to manage arrangement necessity in OSNs to manage the extended requirements of the filtering field. Absolutely, the industry with filtering of disposed of substances instead of with get to control, one of the key essentials frameworks are the openness of a portrayal for the message substance to misuse the sifting strategy.

\section{A. Architecture of Filtered Message}

The design in help of OSN administrations is a three level case. The principle layer as a general rule intends to offer the essential OSN functionalities. OSNs offer a further layer allowing the assistance of outside Social Network Applications (SNA). The maintained SNA may need an additional layer for their required graphical UIs (GUIs). As indicated by the bearing layered basic arrangement, the planned framework must be arranged in the 2nd and 3rd layers in Figure 1. Especially, clients help with the framework utilizing a GUI area up their separating laws, alongside which messages must be sifted through. In the count, the GUI offers clients with a FW that is a divider where essentially messages that are authentic as indicated by their sifting rules are accessible. The central fragments of the methodology are the Content Based Messages Filtering (CBMF) and the Short Text Classifier fundamentals. Then again, the main segments mishandle the message course of action offered the STC component to apply FRs particular by the customer

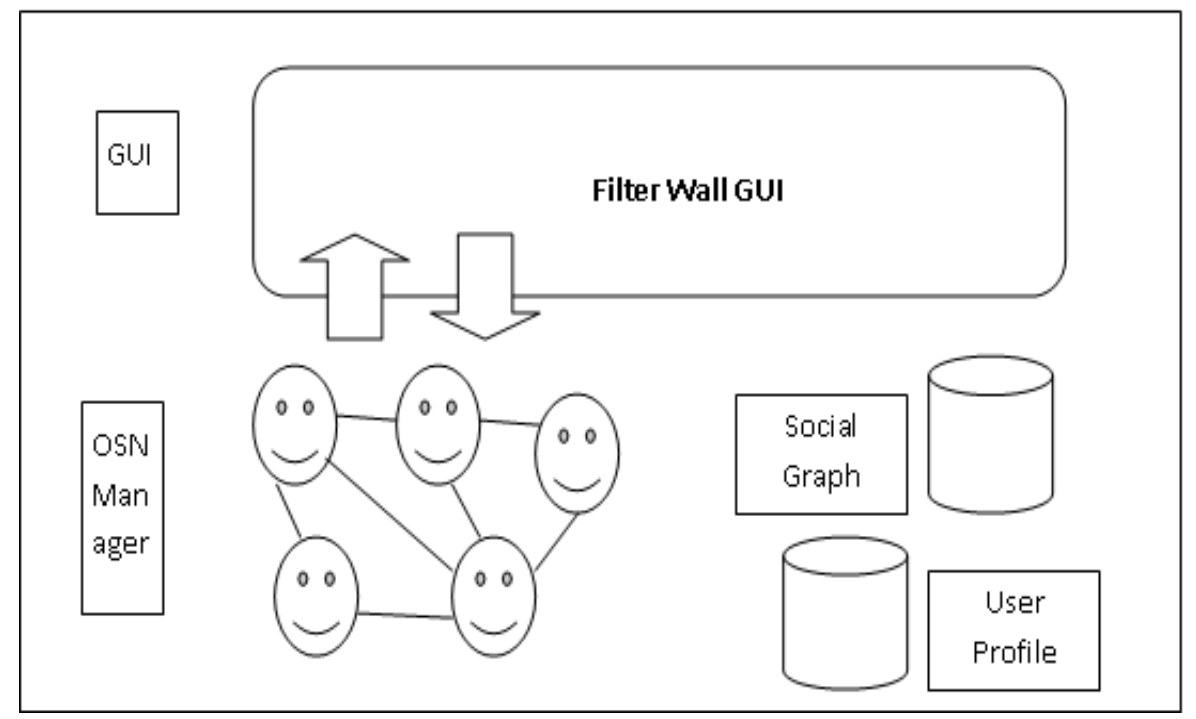

Fig. 1 Workflow of proposed methodology Unwanted filtered message

As diagrammatically outlined in Figure 1, the path required after by a message, it can be abridging as takes after:

$>$ Following entering the private amount of one of his/her organizations, the users endeavors to send a message, which is identified by FW.

$>$ An ML-based data classifier improves metadata from the material. 
$>$ FW utilizes metadata that given by the classification techniques, similarly with blacklisted data from the social diagram and users' profiles, to apply the separating and BL rules.

$>$ Based on the consequence of the previous stride, the content will be accessible or sifted by FW.

\section{B. Short Text Classifier}

Recognized techniques is utilized for content characterizations work well on datasets with vast archives, for example, newswires corpora however endure when the qualifications in the measure are little. In the perspective basic components are the depiction of an arrangement of describing and separated elements permitting the representation of basic ideas and the collection of an entire and dependable arrangement of administered illustrations. The proposed strategy is planning and assessing different portrayal strategies in mix with a neural learning way to deal with semantically order short messages.

\section{Blacklist and Managing Filtering Rules}

Here, the rules taken adopted for filtering discarded the messages. In essential the dialect for sifting laws essential. The strategy considers three fundamental worries that, in induction, should specialist the sifting evaluation.

\section{C.1 Filtering Rules}

A filtering rule FR is a tuple (activity, creator, contentSpec, creatorSpec), where, the creator is the user who differentiates the lead; creatorSpec is a producer particular. contentSpec is a Boolean expression characterized by data limitations of the size $(\mathrm{C}, \mathrm{ml})$. $\mathrm{C}$ is a classification level of the first or second group, and $\mathrm{ml}$ is the base relationship level limit, where fundamental of class $\mathrm{C}$ is to make the confinement fulfilled. Activity $€$ \{block, intimation\} mean the activity to be done by the framework on the content indistinguishable contentSpec and planned by user differentiation by creatorSpec.

The filtering rule is applied for the comparable client. A content is accessible just in the event that it is hindered by any of the sifting decides that identify with the message designer. In addition, that it might occur with client profile does not hold an incentive for the property eluded by a FR in which container; the proposed design is not ready to jag whether the user profile coordinates the FR. Since, how to harmony with such type of content rely upon the deliberate status and the wall holder methods. It claim the divider proprietor to incline toward whether to round or inform content beginning from a user whose profile does not coordinate next to the wall holder FRs since of misleader.

\section{C.2 Blacklists}

A module of proposed design is a BL (Black List method to circumvent content from undesired initiators, free from their substances. BLs straightway supervised by the system. It might be capable to institute whose are introduced in the BL \& select when users' retention in the BL is executed. To advance feasibility, such content are offered to the proposed system through a set of rules, after it called BL rules. Such rules are not distinct by the SNMP; thus, they are not intended as common advanced commands to be convenient for the entire people. Rather, It permit the users themselves, i.e., the wall's holders to allocate $\mathrm{BL}$ rules adaptable who has to be banned from their messages and for how 
extended. As a result, a user might be eliminated from a wall, during matching time by being capable to send in other contents.

A BL rule is a tuple (author, creatorBehavior, creatorSpec, T), wherever

- Novelist is the OSN user who identifies the rule, like wall holder;

- CreatorSpec is a condition maker,

- CreatorBehavior contains of two methods RFBlocked \& minBanned. RFBlocked $=(\mathrm{RF}$, mode, , window $)$ is clear.

- RF $1 / 4$ \#bMessages/\#tMessages, where \#tMessages is the total number of message contents where each OSN users recognized by creatorSpe. They have tried to post in the wall owner (mode $1 / 4$ myWall) or all the OSN conent messages (mode $1 / 4 \mathrm{SN}$ ); where \#bMessages is the integer of messages among various profiles in \#tMessages that have been banned.

- The pane is the time period of creation of messages which have to be considered for RF calculation. Where minBanned = (min, mode, window), min is the minimal amount of times in the gap specified in a pane. The OSN registered people known by creatorSpec, which have to be stored into the $\mathrm{BL}$ due to $\mathrm{BL}$ rules single by Owner wall (mode $=$ myWall) or all OSN users $($ mode $=\mathrm{SN}$ ) to fulfill the criteria.

- T represents the time fazes the user's familiar by creatorSpec and creatorBehavior have to excluded from owner wall.

\section{RESULTS AND DISCUSSION}

\section{A. Programming Environment}

To evaluate the proposed system with existing techniques, the experiments were conducted with Intel i5 Processor with 8 GB RAM running with windows 8, JDK 1.8[12], Netbeans 8.0.2[11], Apache Tomcat 8.0.15[14], and MYSQL 5.7[13] database. To evaluate proposed system with existing method, the method evaluated with Online Social Networks (OSN) database with open source Weka 3.8 library [15].

\section{B. Programming Environment}

The examination of linked work has emphasized the various existing benchmark to evaluate many closest existing methods. It is content-based classification for short texts in OSN. To fulfill the solution of above problems, the system designed a database D of messages content. 1266 messages are collected from publicly available groups extracted using an automatic process which eliminates unwanted jagged messages and, for every message. The method stores in the message body and the forename of the community from which it creates. Table 1 explains the classified result of short text whose details are given in below. The messages approach from the group's web forum, where registered user can post a message or response to users. The set of classes evaluated in the research evaluations is $\Omega=\{$ Neutral; Violence; Vulgar; Offensive; Hate; Sex $\}$, where $\Omega$ $\{$ Neutral\} are the second level classes. 
TABLE 1 The classification Result of Proposed Model

\begin{tabular}{|c|c|c|c|c|c|c|c|}
\hline Metric & Neutrals & $\begin{array}{c}\text { Non- } \\
\text { Neutrals }\end{array}$ & Violence & Vulgar & Offensive & Hate & Sexual \\
\hline P & $81 \%$ & $77 \%$ & $82 \%$ & $62 \%$ & $82 \%$ & $65 \%$ & $88 \%$ \\
\hline R & $93 \%$ & $50 \%$ & $46 \%$ & $49 \%$ & $67 \%$ & $39 \%$ & $91 \%$ \\
\hline F1 & $87 \%$ & $61 \%$ & $59 \%$ & $55 \%$ & $74 \%$ & $49 \%$ & $89 \%$ \\
\hline
\end{tabular}

According to Table 1, it's observed that proposed systems classified the text in following categories, namely: Neutral, Non-Neutral, Violence, Vulgar, and Offensive, Hate, and Sexual words to indentify the unwanted contents in Online Social Networks (OSN). Here, classification result is computed with Precision (P), Recall (R) and F1 Score (F1) where, it notice that Proposed approach predict good accuracy category wise. Figure 2 shows the unwanted content classification results.

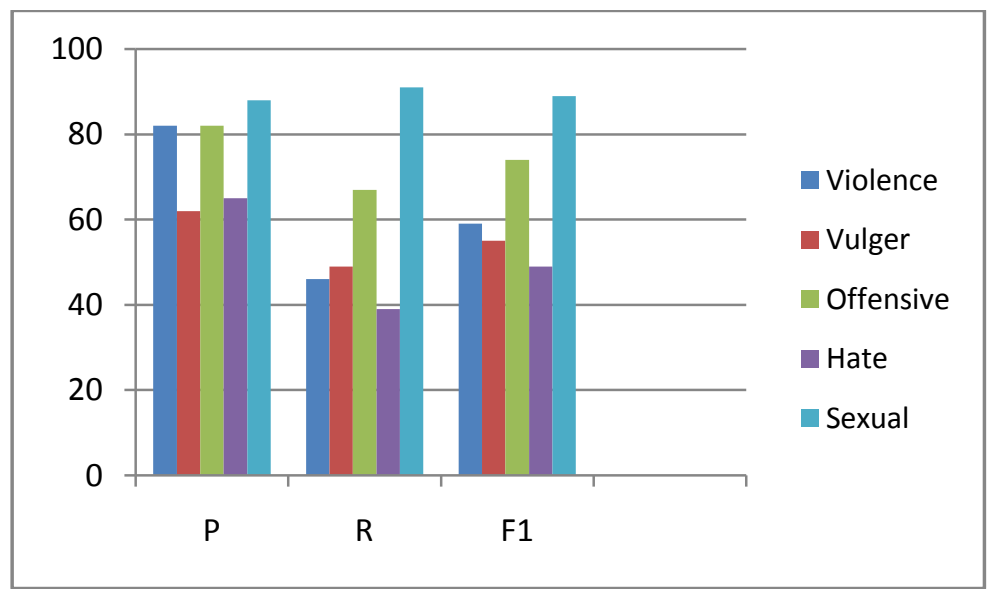

Fig. 2 Proposed approach unwanted content classification result

Based on the result performance in Figure 2, the roposed system is evaluated with various type of ONS database with five types of word categorization namely Neutral, Non-Neutral, Violence, Vulgar, Offensive, Hate, and Sexual. According to result of precision, recall, and F1 score, it observed that proposed system produced best results. Finally, it claims that proposed system is best approach on overall dataset with respective parameters.

\section{CONCLUSION}

In this paper, the proposed system presented to filter undesired contents from OSN messages. The proposed method develops an ML soft classifier to execute effective content-reliant FRs. The goal is to investigate a tool bright to suggest trust values for associated user automatically that does not independently identify. It also judge that such a technique should meet expectation appraisal based on user's procedures, performances, and standing in OSN, which might 
occupy enhancing OSN with measurement methods. These assessment based tools complexes with various concerns, like the suggestions an appraisal system may have users' security $\&$ the restrictions on the basis of a probability to audit in their OSNs. Proposed system is evaluated with various type of ONS database with five types of word categorization namely Neutral, Non-Neutral, Violence, Vulgar, Offensive, Hate, and Sexual. According to result of precision, recall, and F1 score, it observed that proposed system produced best results. Finally, it claims that proposed system is best approach on overall dataset with respective parameters. In future, this paper can be extended to utilize HADOOP environment for filter the unwanted content from Live Social Networks.

\section{REFERENCES}

[1]. M. Vanetti, E. Binaghi, B. Carminati, M. Carullo, and E. Ferrari, "ContentBased Filtering in On-Line Social Networks", Proceedings of ECML/PKDD Workshop Privacy and Security Issues in Data Mining and Machine Learning, 2010, pp. 1-9.

[2]. M. Chau, and H. Chen, "A Machine Learning Approach to Web Page Filtering Using Content and Structure Analysis", Decision Support Systems, Vol. 44, No. 2, 2008, pp. 482-494.

[3]. R.J. Mooney, and L. Roy, "Content-Based Book Recommending Using Learning for Text Categorization", Proceedings of $5^{\text {th }}$ ACM Conference on Digital Libraries, 2000, pp. 195-204.

[4]. F. Sebastian, "Machine Learning in Automated Text Categorization," ACM Computing Surveys, Vol. 34, No, 1, 2002, pp. 1-47.

[5]. A. Adomavicius, and G. Tuzhilin, "Toward the Next Generation of Recommender Systems: A Survey of the State-of-the-Art and Possible Extensions", IEEE Transaction on Knowledge and Data Eng, Vol. 17, No. 6, 2005, pp. 734-749.

[6]. G. Amati, and F. Crestani, "Probabilistic Learning for Selective Dissemination of Messages", Messages Processing and Management, Vol. 35, No. 5, 1999, pp. 633-654.

[7]. P.J. Denning, "Electronic Junk," Comm. ACM, Vol. 25, No. 3, 1982, pp. 163-165.

[8]. P.W. Foltz, and S.T. Dumais, "Personalized Messages Delivery: An Analysis of Messages Filtering Methods," Communication ACM, Vol. 35, No. 12, 1992, pp. 51-60.

[9]. Y. Zhang, and J. Callan, "Maximum Likelihood Estimation for Filtering Thresholds," Proceedings of 24th Annual International ACM SIGIR Conference of Research and Development in Messages Retrieval, 2001, pp. 294-302.

[10]. G. Prakash, B, Vyas, and V.R. Kethu, "Secure \& Efficient Audit Service Outsourcing For Data Integrity In Clouds", International Journal of MC Square Scientific Research, Vol. 6, No. 1, 2014, pp. 50-60.

[11]. https://netbeans.org/downloads/

[12]. https://www.java.com/

[13]. www.mysql.com/downloads/

[14]. http://tomcat.apache.org/

[15]. http://www.cs.waikato.ac.nz/ml/weka/downloading.html 